\title{
Image correlators using optical memory disks
}

\author{
Demetri Psaltis, Mark A. Neifeld, and Alan Yamamura \\ Department of Electrical Engineering, California Institute of Technology, Pasadena, California 91125
}

Received November 14, 1988; accepted February 17, 1989

Image correlators are described and experimentally demonstrated that are implemented using optical memory disks to store a large library of reference images.

Optical correlation for pattern recognition ${ }^{1}$ has long been considered a promising application for optical processing. One of the reasons such correlators have not been used in practice is that in many practical applications a single filter is not sufficient to produce reliable recognition. A straightforward solution to this problem is the use of spatial ${ }^{2}$ and temporal ${ }^{3}$ multiplexing to search through a library of filters. The optical-disk correlator architectures that we describe in this Letter provide an extremely efficient method for performing such a search since they combine in a single device the large memory required for storage of the library of reference images, the spatial light modulator needed to represent the reference in the optical correlator, and the scanning mechanism to search temporally through the library.

The first architecture that we describe is shown in Fig. 1. Each reference image is recorded as a twodimensional (2-D) computer-generated Fourier-transform hologram (CGH) on the disk. The input image enters the system through the beam splitter, is Fourier transformed by the lens, and illuminates the hologram on the disk. The reflected light contains a term proportional to the product of the transforms of the input and reference images. The same lens retransforms the reflected light, and the correlation is produced at the output plane. An important question in this architecture is whether optical disks are suitable as holographic recording media. Figure 2 shows the diffraction pattern obtained with $\mathrm{He}-\mathrm{Ne}$ laser light from a write-once disk manufactured by Sony on which we have recorded a 2-D grating. The sharpness of the characteristic diffraction pattern indicates that the glass cover of the disk has sufficient optical quality to allow coherent reconstruction. The rotation of the disk is used to perform a search through images centered at the same radial position on the disk. An auxiliary scanning mechanism is needed in order to position the correlator head in the correct radial position. As the disk rotates it produces a correlation pattern at the output when the transform of the input and the reference hologram on the disk are in alignment.

The above architecture requires storage of the reference images in the form of computer-generated Fourier-transform holograms. A disadvantage of this approach is that it increases the computational overhead for recording the disk. Also, for a Lohmann-type computer-generated hologram the space-bandwidth product required to record the hologram is one hundred times greater than that of the image itself, and the resultant increase in area needed to record each image increases the optical power and phase uniformity requirements. However, in many cases it is necessary only to record reference holograms as binary patterns, ${ }^{4}$ in which case each pixel of the image can be directly recorded as a separate spot on the disk. Grayscale images can be recorded if necessary by the use of some form of area modulation, as is done with video disks, for example.

We discuss two types of architecture that allow the reference images themselves, rather than their Fourier transforms, to be stored on the disk. The first is shown in Fig. 3. The input image goes through the beam splitter and is Fourier transformed by lens L1. A Fourier-transform hologram of the input is recorded in a photorefractive crystal, using a reference beam that is incident from the right. Once the hologram is recorded the input is blocked and the disk is illuminated. Lens L1 takes the Fourier transform of the reference image that is in the field of view of the illuminating beam, and lens L2 transforms the light diffracted by the hologram to produce the correlation at the output plane. If a thick hologram is used, the shift

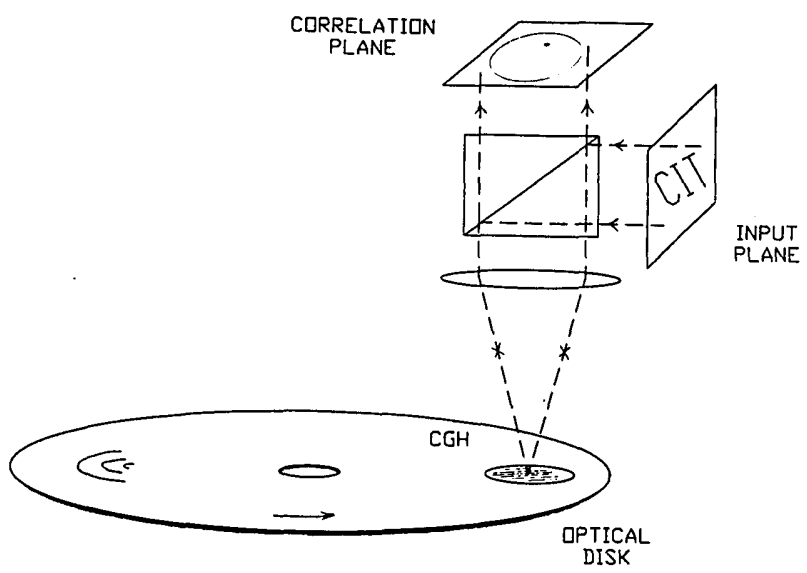

Fig. 1. Optical disk-based Vander Lugt correlator. The reference images are stored on the disk as computer-generated Fourier-transform holograms. 


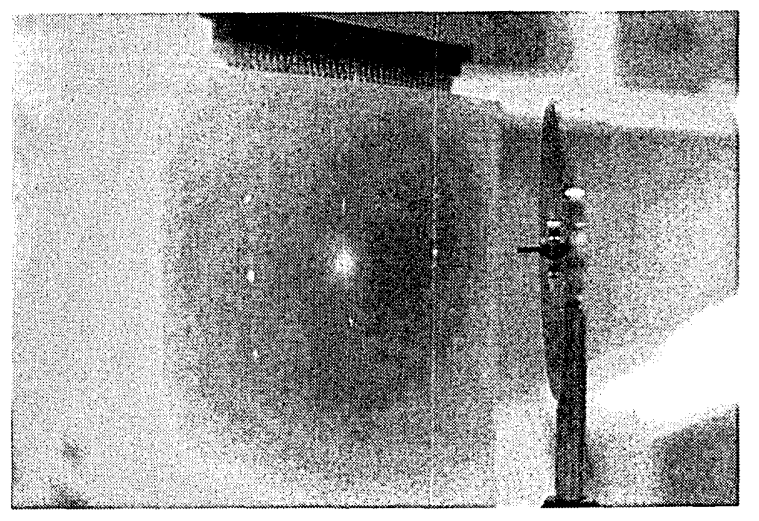

Fig. 2. Diffraction pattern from a 2-D grating recorded on a write-once optical disk.

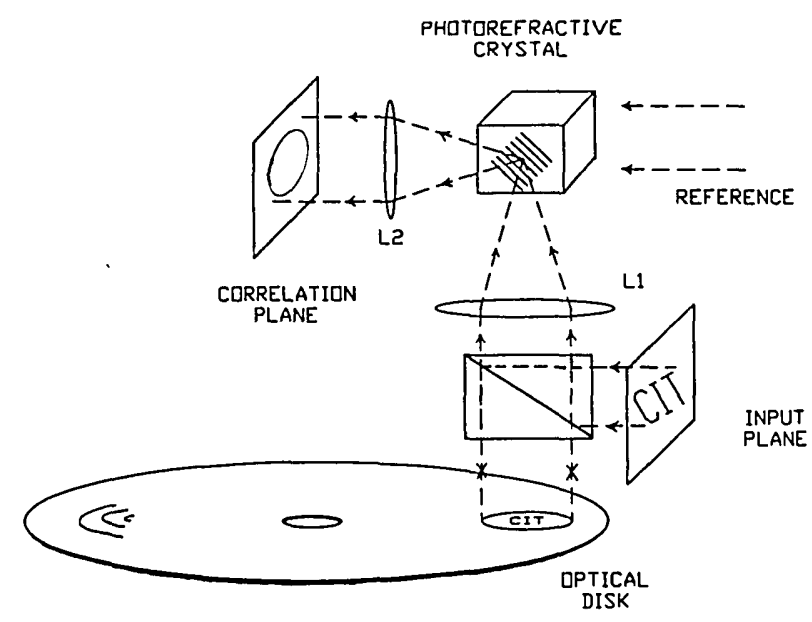

Fig. 3. Optical disk-based correlator implemented by using a photorefractive crystal in the Fourier plane.

invariance is lost in one direction only. ${ }^{5}$ Selecting this to be the along-track direction allows the disk rotation to restore 2-D shift invariance. The rotation of the disk is used to search through a library of images in the along-track direction. An advantage of this architecture is that it is invariant to a shift in the position of the recording on the disk. This eliminates the requirement for precise alignment of the correlator head, and in addition a time-delay-and-integrate detector can be used to integrate the traveling correlation pattern at the output, thereby increasing sensitivity. Multiple holograms could be recorded in the crystal to address different radial positions on the disk, or the entire head can be mechanically scanned.

The third architecture that we discuss is shown schematically in Fig. 4. The advantage of this architecture is that it operates on the light intensity, and consequently the requirement for phase uniformity is greatly relaxed. ${ }^{6}$ As a result it is possible to implement this architecture with most existing disk systems. This correlator works as follows. The reference images are recorded on the disk, and the input is imaged through a one-dimensional scanning device onto the disk. The scanner can be either an acousto- optic device (AOD) (as shown in Fig. 4) or a rotating mirror. It provides the relative displacement in the radial direction between the input and reference images that is necessary to calculate the correlation function. The disk rotation provides the displacement in the orthogonal direction. The scanner translates the input image completely across the stored reference image each time the disk rotates by a distance equal to a pixel of the reference. The intensity of the light reflected from the disk at any one time is proportional to the product of the input and a shifted version of the reference. The reflected light is collected (integrated) on a single detector that produces as its output a temporal video signal of the 2-D correlation. This system was experimentally demonstrated with a flying-spot acousto-optic scanner in which a chirp signal propagates in the acousto-optic device acting as a traveling lens that scans the diffracted image at a rate equal to the acoustic velocity. This system completes a scan in $30 \mu \mathrm{sec}$ (the acoustic transit time across the acoustooptic cell), therefore a complete 2-D correlation of an image consisting of $10^{2}$ lines takes approximately 3 msec. A sample of experimental results obtained with a system like that of Fig. 4 is shown in Fig. 5. Figure 5 (a) is a photograph of the pattern recorded on a writeonce disk (the acronym CIT), and Fig. 5(b) is an oscilloscope trace of the detector signal produced by the optical system of Fig. 4. Figure 5(c) shows the same trace magnified to reveal the individual correlation lines produced by the acousto-optic scanner. The format of the detector signal is similar to a video signal of the 2-D correlation, and it can be displayed in two dimensions by raster scanning the detector output on a 2-D monitor [Fig. 5(d)]. Correlations can be produced with our experimental apparatus at rates up to $1000,100 \times 100$ pixel reference images/sec. The optically calculated correlation is in good agreement with the expected autocorrelation function of the CIT pattern. It should be pointed out that since this system operates on intensity, only positive quantities can be represented. Bipolar input and/or reference images can also be represented by adding a bias at the input stage and subtracting it from the output. This tech-

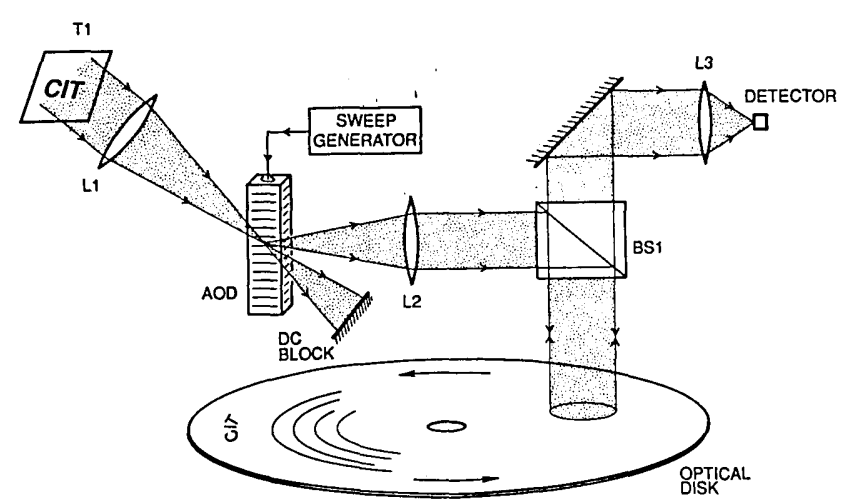

Fig. 4. Incoherent disk-based optical correlator implemented with an acousto-optic scanner. T1, input transparency; L's, lenses; BS1, beam splitter. 


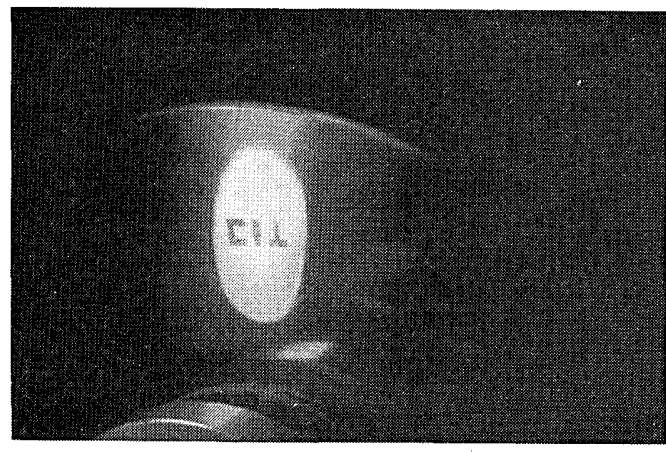

(a)

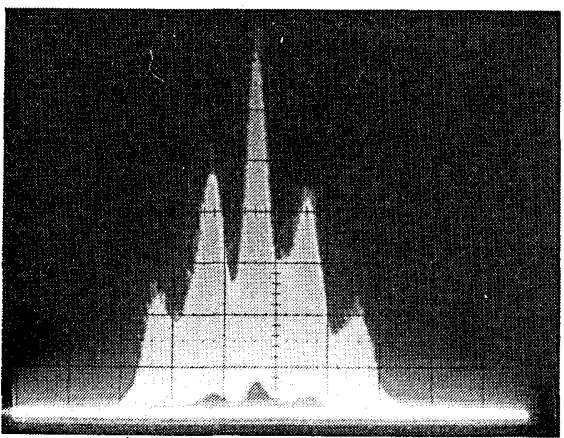

(b)

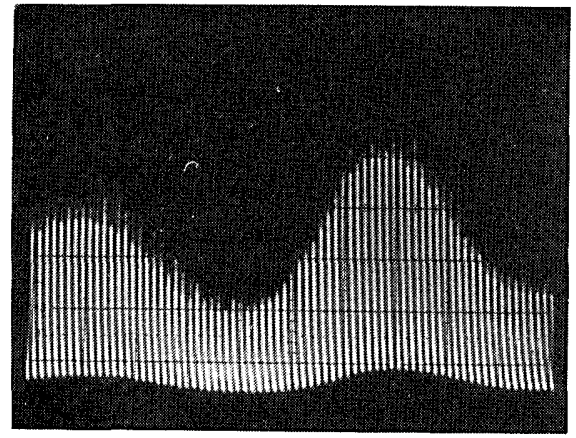

(c)

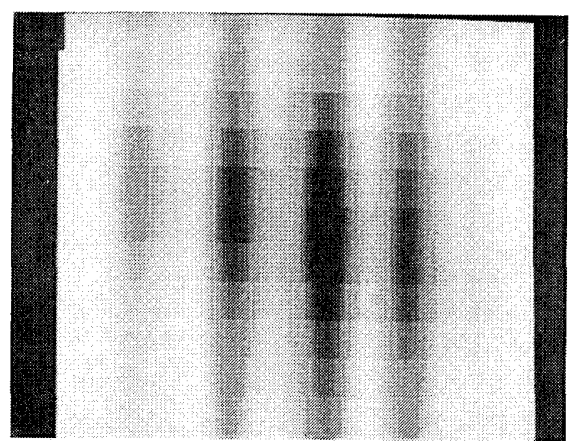

(d)

Fig. 5. Experimental demonstration of the system shown in Fig. 4. (a) The pattern recorded on the optical disk. (b) An oscilloscope trace of the detector output. (c) A magnified version of the signal shown in (b) revealing the correlations of the individual lines. (d) The 2-D correlation produced by raster recording the signal shown in (b).

nique has been successfully used in a variety of incoherent architectures. ${ }^{3}$

More than 5 billion bits can be stored in the type of disk that we use for most of our research (a write-once, 12-cm-diameter system from Sony). The number of $100 \times 100$ pixel images that can be stored in such a disk is more than 5000 , if we assume a generous factor of 100 for loss of space-bandwidth product owing to representation (e.g., area modulation for gray-scale representation). The rate at which all these images can be interrogated for a possible match with the input is limited by one or more of the following factors: the scanning speed of the disk $(40 \mathrm{~Hz}$ in our case), the speed of the radial scanning mechanism, and the sensitivity and the bandwidth of the output detectors and the electronics following them. As an example consider the system of Fig. 2. At a $40-\mathrm{Hz}$ disk rotation rate we obtain 1000 image correlations per $1 / 40$ of a second (i.e., 40,000 image correlations/sec), which yields a reasonable $4-\mathrm{MHz}$ bandwidth per detector. The input optical power required for reliable detection of the correlation peak is only several milliwatts. It would be extremely difficult to duplicate this capability electronically, and it can be achieved with existing optical technology. Moreover it is precisely such capability that is required for practical pattern-recognition problems.

The research reported in this Letter is supported by the U.S. Army Research Office.

\section{References}

1. A. Vander Lugt, IEEE Trans. Inf. Theory IT-10, 2 (1964).

2. B. D. Guenther, C. R. Christensen, and J. Upatnieks, IEEE J. Quantum Electron. QE-15, 1348 (1979).

3. D. Psaltis, Opt. Eng. 23, 1 (1984).

4. D. Psaltis, E. G. Paek, and S. S. Vankatesh, Opt. Eng. 23, 698 (1984).

5. J. W. Yu, F. H. Mok, and D. Psaltis, Proc. Soc. Photo-Opt. Instrum. Eng. 825, 22 (1987).

6. A. W. Lohmann and H. W. Werlich, Appl. Opt. 10,670 (1971). 